\title{
Universality of particle production and energy balance in hadronic and nuclear collisions
}

\author{
Aditya Nath Mishra ${ }^{1, a}$, Edward K. G. Sarkisyan, ${ }^{2,3, b}$, Raghunath Sahoo, ${ }^{1, \mathrm{c}}$, and Alexander S. \\ Sakharov $2,4,5, \mathrm{~d}$ \\ ${ }^{1}$ Discipline of Physics, School of Basic Science, Indian Institute of Technology Indore, Indore-452017, India \\ ${ }^{2}$ Department of Physics, CERN, 1211 Geneva 23, Switzerland \\ ${ }^{3}$ Department of Physics, The University of Texas at Arlington, Arlington, TX 76019, USA \\ ${ }^{4}$ Department of Physics, New York University, New York, NY 10003, USA \\ ${ }^{5}$ Physics Department, Manhattan College, New York, NY 10471, USA
}

\begin{abstract}
The multihadron production in nucleus-nucleus and (anti)proton-proton collisions is studied by exploring the collision-energy and centrality dependencies of the mean multiplicity in the existing data. The study is performed in the framework of the recently proposed effective-energy approach which combines the constituent quark picture and Landau hydrodynamics counting for the centrality-defined effective energy of participants. Within this approach, the multiplicity energy dependence and the pseudorapidity spectra from the most central nuclear collisions are well reproduced. The study of the multiplicity centrality dependence reveals a new scaling between the measured pseudorapidity spectra and the calculations. Using this scaling, called the energy balanced limiting fragmentation scaling, the pseudorapidity spectra are well reproduced for all centralities. The scaling clarifies some differences in the multiplicity and midrapidity density centrality dependence from RHIC and LHC. A similarity in the multiplicity energy dependence in the most central collisions and centrality data is shown. Predictions are drawn for the mean multiplicities to be measured in hadronic and heavy-ion collisions at the LHC.
\end{abstract}

1. In this report, we discuss our recent results on the universality of multiparticle production in nucleus-nucleus (AA) and hadronic interactions in view of a new scaling obtained [1]. The study exploites concept of effective energy [2] employed for the data interpreted in terms of the approach of the dissipating energy of quark participants [3, 4], or, for brevity, the participant dissipating energy (PDE) approach. This approach combines the constituent quark picture together with Landau relativistic hydrodynamics and interrelates different types of collisions. The earlier observations [2] are made by studying the dependencies of the pseudorapidity density and transverse energy pseudorapidity density at midrapidity on the collision center-of-mass (c.m.) energy in hadronic and the most central (head-on) AA collisions and on the number of nucleon participants, or centrality, in AA collisions in the entire available energy range of the existing data. The complementarity of the measurements in

\footnotetext{
ae-mail: Aditya.Nath.Mishra@cern.ch

be-mail: sedward@cern.ch

ce-mail: Raghunath.Sahoo@cern.ch

de-mail: Alexandre.Sakharov@ cern.ch
} 
non-central and head-on AA collisions is shown. Here, in the framework of the PDE approach, we extend the previous studies of the charged particle mean multiplicity $[3,4]$ to LHC energies.

This approach quantifies the process of particle production in terms of the amount of energy deposited by interacting constituent quark participants inside the small Lorentz-contracted volume. The whole process of a collision is then represented as the expansion of an initial state and the subsequent break-up into particles. This approach resembles the Landau phenomenological hydrodynamic approach of multiparticle production in relativistic particle interactions [5]. In the picture considered here, the Landau hydrodynamics is employed in the framework of constituent (or dressed) quarks, in accordance with the additive quark model [6]. This makes the secondary particle production to be basically driven by the amount of the initial effective energy deposited by constituent quarks. In $p p / \bar{p} p$ collisions, a single constituent quark from each nucleon is considered to take part in a collision and the remaining quarks are treated as spectators. Thus, the effective energy for the production of secondary particles is $1 / 3$ of the entire nucleon energy. On the contrary, in the head-on AA collisions, the participating nucleons are considered colliding with all three constituent quarks from each nucleon. This makes the whole energy of the colliding nucleons (participants) available for the secondary particle production. Within this picture, one expects the results for bulk observables from head-on AA collisions at the c.m. energy per nucleon, $\sqrt{s_{N N}}$, to be similar to those from the $p p / \bar{p} p$ collisions at $\sqrt{s_{p p}} \simeq 3 \sqrt{s_{N N}}$. Such an universality is found to correctly predict [3] the value of the midrapidity density in $p p$ interactions measured at the TeV LHC energies [7].

Combining the above-discussed ingredients, one obtains the relationship between the rapidity density per participant pair, $\rho(\eta)=\left(2 / N_{\text {part }}\right) d N_{\mathrm{ch}} / d \eta$ at midrapidity $(\eta \approx 0)$, in AA and $p p / \bar{p} p$ collisions:

$$
\rho(0) / \rho_{p p}(0)=2 N_{\mathrm{ch}} /\left(N_{\mathrm{part}} N_{\mathrm{ch}}^{p p}\right) \sqrt{L_{p p} / L_{N N}}, \quad \sqrt{s_{p p}}=3 \sqrt{s_{N N}} .
$$

In Eq.(1), the relation of the pseudorapidity density and the mean multiplicity is applied in its Gaussian form as obtained in Landau hydrodynamics. The factor $L$ is defined as $L=\ln (\sqrt{s} / 2 m)$. According to the approach considered, $m$ is the proton mass, $m_{p}$, in AA collisions and the constituent quark mass in $p p / \bar{p} p$ collisions set to $\frac{1}{3} m_{p} . N_{\mathrm{ch}}$ and $N_{\mathrm{ch}}^{p p}$ are the mean multiplicities in AA and nucleon-nucleon collisions, respectively, and $N_{\text {part }}$ is the number of participants.

Solving Eq. (1) for the multiplicity $N_{\mathrm{ch}}$ at a given rapidity density $\rho(0)$ at $\sqrt{s_{N N}}$, and for the rapidity density $\rho_{p p}(0)$ and the multiplicity $N_{\text {ch }}^{p p}$ at $3 \sqrt{s_{N N}}$, one finds:

$$
2 N_{\mathrm{ch}} / N_{\mathrm{part}}=N_{\mathrm{ch}}^{p p} \rho(0) / \rho_{p p}(0) \sqrt{1-2 \ln 3 / \ln \left(4.5 \sqrt{s_{N N}} / m_{p}\right)}, \quad \sqrt{s_{N N}}=\sqrt{s_{p p}} / 3 .
$$

Further development [2] treats this dependence in terms of centrality. The most central collisions correspond to the lowest centrality while the larger centrality to the more peripheral collisions. The centrality is related to the number of participants and, then, to the amount of the energy released in the collisions, i.e., to the effective energy, $\varepsilon_{N N}$. In the framework of the PDE approach, $\varepsilon_{N N}$ can be defined as a fraction of the c.m. energy available in a collision according to the centrality, $\alpha:^{1}$

$$
\varepsilon_{N N}=\sqrt{s_{N N}}(1-\alpha) .
$$

Then, for the effective c.m. energy $\varepsilon_{N N}$, Eq. (2) reads:

$$
2 N_{\mathrm{ch}} / N_{\mathrm{part}}=N_{\mathrm{ch}}^{p p} \rho(0) / \rho_{p p}(0) \sqrt{1-2 \ln 3 / \ln \left(4.5 \varepsilon_{N N} / m_{p}\right)}, \quad \varepsilon_{N N}=\sqrt{s_{p p}} / 3,
$$

\footnotetext{
${ }^{1}$ Conventionally, the data are divided into centrality intervals, so that $\alpha$ is the average centrality per centrality interval, e.g. $\alpha=0.25$ for the centrality interval of $20-30 \%$ centrality.
} 
where $\rho(0)$ is the midrapidity density in central AA collisions measured at $\sqrt{s_{N N}}=\varepsilon_{N N}$.

2. Figure 1(a) shows the c.m. energy dependence of the multiplicity measured in head-on AA collisions [8-13] and the fits made in the energy range of $\sqrt{s_{N N}}=2 \mathrm{GeV}$ to $2.76 \mathrm{TeV}$. Given the fact that the measurements support the 2nd-order logarithmic dependence on $\sqrt{s_{N N}}$ up to the top RHIC energy $[3,10]$ while the power-law dependence is obtained for the LHC data [8], we fit the head-on data by the "hybrid" fit function. In addition to the hybrid fit, we show the $\log ^{2}\left(s_{N N}\right)$-fit $[3,4]$ up to the top RHIC energy and the power-law fit. One can see that the power-law fit well describes the data and is almost indistinguishable from the hybrid fit up to the LHC data. However, the 2nd-order logpolynomial lies below the data for $\sqrt{s_{N N}}>200 \mathrm{GeV}$. This observation supports a possible transition to a new regime in AA collisions at $\sqrt{s_{N N}}$ of about $1 \mathrm{TeV}$, as indicated earlier [2].

Addressing now Eq. (2), one calculates $N_{\mathrm{ch}} /\left(N_{\text {part }} / 2\right)$ for AA interactions using the $p p / \bar{p} p$ data. The $\rho_{p p}(0)$ and $N_{\text {ch }}^{p p}$ values are taken from the existing data [15] or, where not available, calculated using the corresponding experimental $\sqrt{s_{p p}}$ fits ${ }^{2}$ at $\sqrt{s_{p p}}=3 \sqrt{s_{N N}}$. The $\rho(0)$ values are also from the measurements in central AA collisions where available, otherwise the hybrid fit [2] is used.

One can see that the calculated $N_{\mathrm{ch}} /\left(0.5 N_{\text {part }}\right)$ values follow the measurements from AA collisions at $\sqrt{s_{N N}}$ from a few $\mathrm{GeV}$ up to the TeV LHC energy. This points to the universality of the multiparticle production in the different types of collisions.

Solving Eq. (2) for $N_{\mathrm{ch}}^{p p}$, one estimates [1] its values to be about 48 at $\sqrt{s_{p p}}=2.36 \mathrm{TeV}, 69$ at 7 $\mathrm{TeV}$, and 79 at $13 \mathrm{TeV}$ with 5 to $10 \%$ uncertainties. The study [1] shows that the $\log ^{2}(s)$-polynomial fit function is very close to the power-law function in the c.m. energy range from a few $\mathrm{GeV}$ up to about a few TeV, similar to the earlier observations for $\sqrt{s_{p p}}>53 \mathrm{GeV}$ [14]. The $\log ^{2}(s)$-polynomial function is not far from the power-law fit even for $\sqrt{s_{p p}}>2 \mathrm{TeV}$. This may point to apparently no change in the multihadron production in $p p$ interactions up to the highest LHC energy, in contrast to a new regime possibly emerging at $\sqrt{s_{N N}} \approx 1 \mathrm{TeV}$ in AA collisions.

In Fig. 1(b), we show the $N_{\text {part }}$-dependence of $N_{\text {ch }} /\left(N_{\text {part }} / 2\right)$. One can see that the $\varepsilon_{N N}$-calculations well reproduce the LHC data except slightly underestimating a couple of the most peripheral measurements. For the RHIC data, however, the difference between the calculations and the measurements is visible already for medium centralities, i.e., for more central collisions. These observations are also interrelated with the difference observed in the measurements at RHIC vs. those from LHC. This becomes even clearer when the $200 \mathrm{GeV}$ PHOBOS data are multiplied by a factor of 2.87 which allows matching the ALICE data from the highly central collisions.

To clarify the observed differences, in the following sections the distributions of the pseudorapidity density are investigated in the context of the PDE picture.

3. Fig. 2 shows the distributions $\rho(\eta)$ of charged particles measured in head-on and very central AA collisions and $\rho(\eta)_{p p}$ in $p p / \bar{p} p$ interactions at $\sqrt{s_{p p}} \approx 3 \sqrt{s_{N N}}$ or $3 \varepsilon_{N N}$.

Within the considered model of constituent quarks and the Gaussian form of the pseudorapidity distribution in Landau hydrodynamics, the relationship between $\rho(\eta)$ and $\rho_{p p}(\eta)$ reads

$$
\rho(\eta) / \rho_{p p}(\eta)=2 N_{\mathrm{ch}} /\left(N_{\text {part }} N_{\mathrm{ch}}^{p p}\right) \sqrt{1+2 \ln 3 / L_{N N}} \exp \left[-\eta^{2} /\left(L_{N N}\left(2+L_{N N} / \ln 3\right)\right)\right] .
$$

The calculations are shown in Fig. 2 and demonstrate very good agreement with the measurements. Minor deviations are due to some mismatch between $\sqrt{s_{p p}}$ and $3 \sqrt{s_{N N}}$ (or $3 \varepsilon_{N N}$ ) and, as expected, due to a slight non-centrality. It is noticeable how well the PDE picture allows one to reproduce the pseudorapidity density distributions in AA interactions in the full- $\eta$ range, from central- $\eta$ to forward- $\eta$

\footnotetext{
${ }^{2}$ The hybrid fit [1] is used for $N_{\mathrm{ch}}^{p p}$, while $\rho_{p p}(0)$ is calculated using the linear-log fit $\rho_{p p}(0)=-0.308+0.276 \ln \left(s_{p p}\right)$ [14] and the power-law fit by CMS [16], $\rho_{p p}(0)=-0.402+s_{p p}^{0.101}$, at $\sqrt{s_{p p}} \leq 53 \mathrm{GeV}$ and at $\sqrt{s_{p p}}>53 \mathrm{GeV}$, respectively.
} 


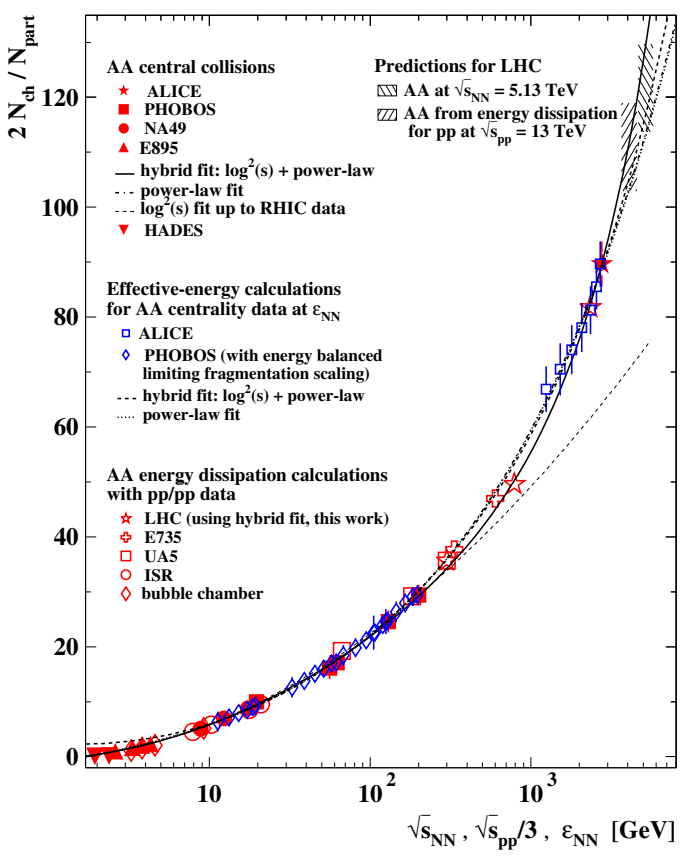

a)

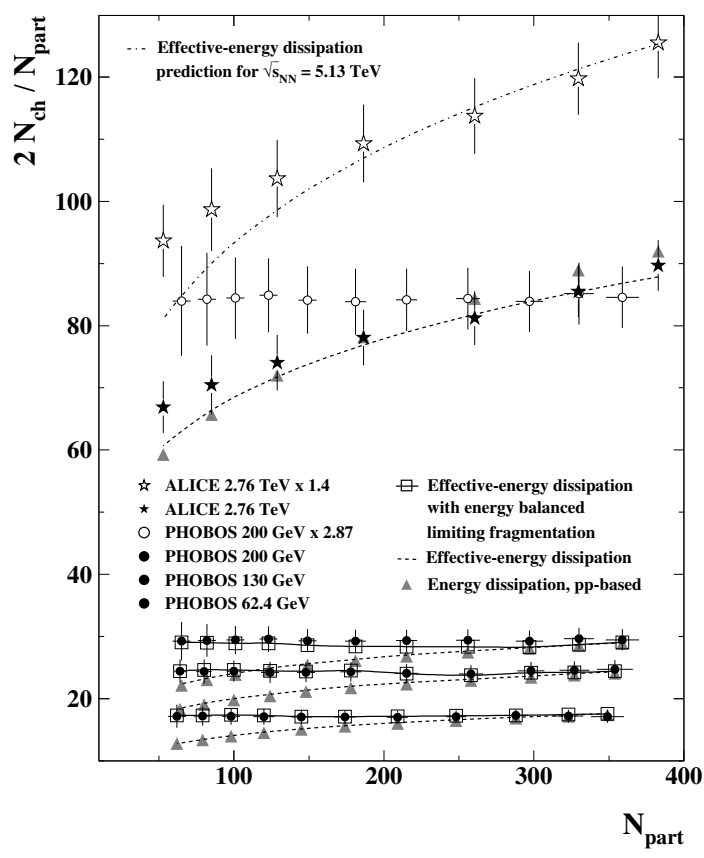

b)

Figure 1. (a) The energy dependence of the charged particle mean multiplicity per participant pair. The large solid symbols show the measurements from the most central nucleus-nucleus (AA) collisions [8-13] given as a function of the nucleon-nucleon c.m. energy, $\sqrt{s_{N N}}$. The calculations by Eq. (2) based on $p p / \bar{p} p$ data [14, 15] at the c.m. energy $\sqrt{s_{p p}}=3 \sqrt{s_{N N}}$ are shown vs. $\sqrt{s_{p p}} / 3$ by large open symbols. The small open symbols show the AA data at different centralities by using Eq. (4) at the effective energy $\varepsilon_{N N}$ (Eq. (3)). The RHIC centrality data are shown after removing the energy balanced limiting fragmentation scaling ingredient from the calculations of Eq. (4), while the calculations do not take into account this ingredient for the LHC centrality data (see text). The LHC multiplicities in $p p$ interactions are calculated using the hybrid fit from [1]. The solid and the dasheddotted lines show, correspondingly, the hybrid fit, $-0.577+0.394 \ln \left(s_{N N}\right)+0.213 \ln ^{2}\left(s_{N N}\right)+0.005 s_{N N}^{0.551}$, and the power-law fit, $-6.72+5.42 s_{N N}^{0.181}$, to the most central AA data. The thin dashed line shows the 2nd-order log-fit $-0.35+0.24 \ln \left(s_{N N}\right)+0.24 \ln ^{2}\left(s_{N N}\right)$ to the most central AA data up to the top RHIC energy [3, 4]. The dashed and the dotted lines show, correspondingly, the hybrid fit, $2.45-1.06 \ln \left(\varepsilon_{N N}\right)+1.04 \ln ^{2}\left(\varepsilon_{N N}\right)+0.082 \varepsilon_{N N}^{0.744}$, and the power-law fit, $-6.55+5.39 \varepsilon_{N N}^{0.362}$, to the centrality AA data. The right-inclined hatched area shows the prediction for AA collisions at $\sqrt{s_{N N}}=5.13 \mathrm{TeV}$ and the left-inclined hatched area gives the prediction expected from $p p$ collisions at $\sqrt{s_{p p}}=13 \mathrm{TeV}$. (b) The charged particle mean multiplicity per participant pair as a function of the number of participants, $N_{\text {part }}$. The solid circles show the dependence measured in AuAu collisions at RHIC by the PHOBOS experiment at $\sqrt{s_{N N}}=62.4,130$ and $200 \mathrm{GeV}$ [10] (bottom to top). The solid stars show the measurements from PbPb collisions at the LHC by the ALICE experiment at $\sqrt{s_{N N}}=2.76 \mathrm{TeV}$ $[8,17]$. The triangles show the calculations by Eq. (4) using $p p / \bar{p} p$ data. The lines represent the calculations within the effective-energy approach based on the hybrid fit obtained for the c.m. energy dependence of the mean multiplicity in the most central AA collisions shown in (a). The open squares show the effective-energy calculations which include the energy balanced limiting fragmentation scaling (see text); the solid lines connect the calculations to guide the eye. The open circles show the PHOBOS measurements at $\sqrt{s_{N N}}=200 \mathrm{GeV}$ multiplied by 2.87 . The open stars show the ALICE measurements at $\sqrt{s_{N N}}=2.76 \mathrm{TeV}$ multiplied by 1.4 . 


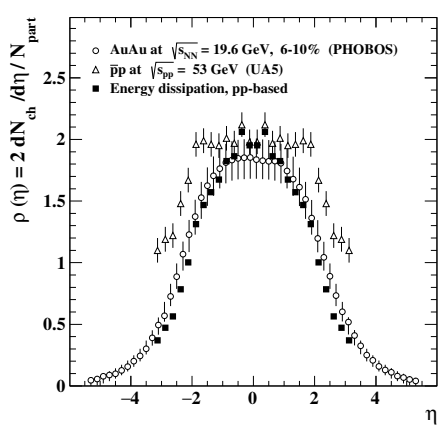

a)

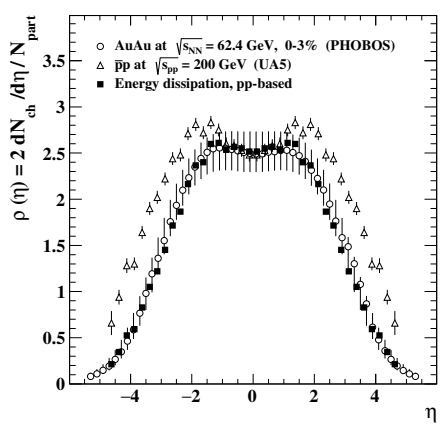

b)

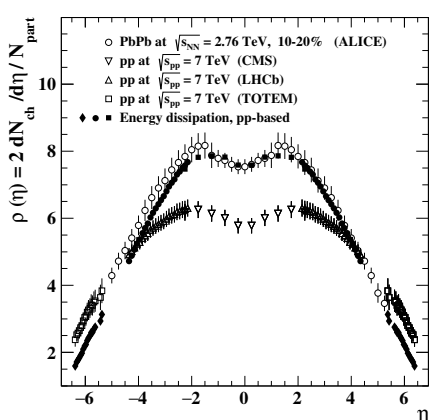

c)

Figure 2. The pseudorapidity distributions of charged particle pseudorapidity density per participant pair. The open circles show the measurements at RHIC at (a) $\sqrt{s_{N N}}=19.6 \mathrm{GeV}$, (b) $62.4 \mathrm{GeV}$ [10], and (c) at the LHC at $\sqrt{s_{N N}}=2.76 \mathrm{TeV}$ [8]. The open triangles show the distributions measured in $p p / \bar{p} p$ interactions at $\sqrt{s_{p p}}=$ $53 \mathrm{GeV}$ to $7 \mathrm{TeV}[15,18,19]$. The solid markers show the calculations by Eq. (5) using $p p / \bar{p} p$ data at $\sqrt{s_{p p}} \approx$ $3 \sqrt{s_{N N}}$ or $3 \varepsilon_{N N}$. Apart from the CMS data, the negative- $\eta$ data points for $p p / \bar{p} p$ interactions are the reflections of the measurements taken in the positive- $\eta$ region.

regions, in the $\sqrt{s_{N N}}$ range spanning over more than two orders of magnitude and for different relative heights of AA vs. $p p$ spectra.

Let us now address peripheral collisions to clarify the deviation between the data and the calculations in Fig 1(b).

In Fig. 3(a) the distributions $\rho(\eta)$ measured [10] in AuAu collisions at $\sqrt{s_{N N}}=130 \mathrm{GeV}$ at 45 $50 \%$ centrality, $\alpha=0.475$, and in $\bar{p} p$ collisions at $\sqrt{s_{p p}}=200 \mathrm{GeV}$ [15], i.e., at $\sqrt{s_{p p}} \approx 3 \varepsilon_{N N}$. The $\rho(\eta)$ spectrum, calculated by Eq. (5), is also shown in Fig. 3(a) and provides a good agreemnet with the measurements in the central- $\eta$ region while fall below the data outside this region. This finding shows that in non-central collisions, the calculations within the PDE approach reproduces well the pseudorapidity density around the midrapidity while underestimate the mean multiplicity. The former conclusion is well confirmed by our recent studies reported in [2] for the midrapidity observables, and the latter one is demonstrated by Fig. 1(b).

The following comments are in order.

In the PDE picture, the global observables are defined by the energy of participants, deriving the bulk production by the initial energy deposited at zero time at rapidity $\eta=0$, similar to the Landau hydrodynamics. Then, as it is expected and just commented, the pseudorapidity density at midrapidity is well reproduced for all types of AA collisions, from the most central to peripheral ones.

From Fig. 3(a), one can see that the calculated distribution $\rho(\eta)$ is narrower than that of the data. This is explained by a smaller value of $\varepsilon_{N N}$ compared to the value of the actual collision energy $\sqrt{s_{N N}}$ while the calculations in Eq. (5) are made with the multiplicity $N_{\text {ch }}$ taken from the most central AA collisions at the c.m. energy equal to $\varepsilon_{N N}$. In other words, in the approach applied here, similar to the Landau hydrodynamics, AA collisions are treated head-on-like.

4. It is established that at high enough energies, in different types of interactions the pseudorapidity density spectra, measured at different c.m. energies, become similar in the fragmentation region. It means that they are independent of a projectile state for the same type of colliding objects, i.e. being 


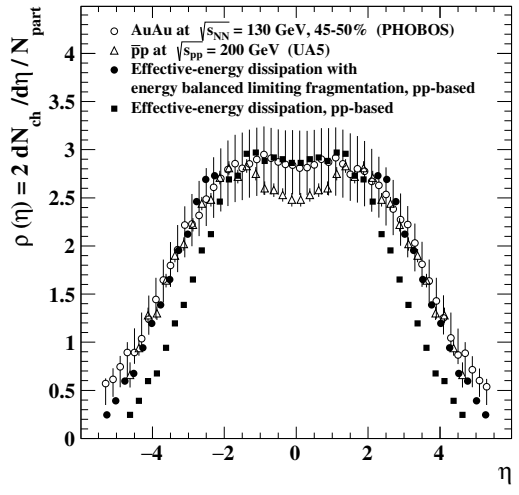

a)

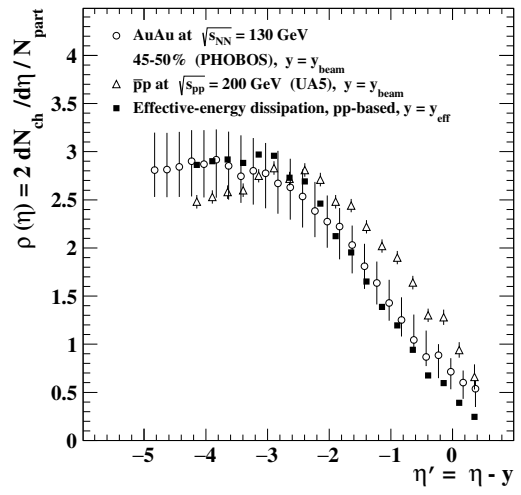

b)

Figure 3. (a) The charged particle pseudorapidity density distributions per participant pair. The open markers show the data from AuAu collisions [10] and $\bar{p} p$ interactions [15]. The solid squares show the distribution calculated from Eq. (5). $\sqrt{s_{p p}} \approx 3 \varepsilon_{N N}$ (see Eq. (3) for the definition of $\varepsilon_{N N}$ ). The solid circles show the beyondmidrapidity part obtained from the calculations using the energy balanced limiting fragmentation scaling, i.e. under the shift $\eta \rightarrow \eta-\ln \left(\varepsilon_{N N} / \sqrt{s_{N N}}\right)$. The negative- $\eta$ data points for $\bar{p} p$ interactions are the reflections of the measurements taken in the positive- $\eta$ region. (b) Same as (a) but the measured distributions of AuAu and $\bar{p} p$ collisions are shifted by the beam rapidity, $\eta^{\prime}=\eta-y_{\text {beam }}$, with $y_{\text {beam }}=\ln \left(\sqrt{s} / m_{p}\right)$, where $s=s_{N N}$ and $s_{p p}$, correspondingly, and the calculated distribution is shifted to $\eta^{\prime}=\eta-y_{\text {eff }}$ with $y_{\text {eff }}=\ln \left(\varepsilon_{N N} / m_{p}\right)$. The shifted distributions - the one measured in AuAu collisions and the calculated one - agree well in the fragmentation region that represents the energy balanced limiting fragmentation scaling.

considered as a function of $\eta^{\prime}=\eta-y_{\text {beam }}$, where $y_{\text {beam }}=\ln \left(\sqrt{s_{N N}} / m_{p}\right)$ is the beam rapidity [14, 20]. This observation obeys a hypothesis of the limiting fragmentation scaling [21].

Considering the limiting fragmentation hypothesis within the effective-energy approach, one expects the limiting fragmentation scaling of the distribution $\rho(\eta)$, which is measured at $\sqrt{s_{N N}}$, to be similar to that of the calculated distribution but taken at the effective energy $\varepsilon_{N N}$.

In Fig. 3(b), the limiting fragmentation hypothesis is applied to both the measured and the calculated pseudorapidity density distributions $\rho(\eta)$ from Fig. 3(a) using the c.m. energy and the effective energy, respectively. Therefore the measured distribution $\rho(\eta)$ is shifted by the beam rapidity, $y_{\text {beam }}$, while the calculated distribution from Eq. (5) is shifted by $y_{\mathrm{eff}}=\ln \left(\varepsilon_{N N} / m_{p}\right)$ and becomes a function of $\eta^{\prime}=\eta-y_{\text {eff }}$. One can see that the calculated $\rho\left(\eta^{\prime}\right)$ distribution of non-central AA collisions agrees well with the measured distribution $\rho\left(\eta^{\prime}\right)$. This finding points to a new energy scaling as soon as the effective-energy approach is applied. In analogy with the limiting fragmentation scaling, we call the observed scaling the energy balanced limiting fragmentation scaling. Due to this scaling, the calculated pseudorapidity density is getting corrected outside the central- $\eta$ region accordingly.

To this end, in Fig. 3(a), the calculated distribution $\rho(\eta)$ is shifted by the difference $\left(y_{\text {eff }}-y_{\text {beam }}\right)$ in this region: $\eta \rightarrow \eta-\ln \left(\varepsilon_{N N} / \sqrt{s_{N N}}\right)$, or, using the effective energy definition, Eq. (3), $\eta \rightarrow \eta-\ln (1-\alpha)$. The shift balances the energy and this brings the calculations to the measured pseudorapidity density distribution in the full- $\eta$ range in non-central AA collision.

This finding allows obtaining $N_{\mathrm{ch}}$ within the PDE approach. Namely, the difference between the two $N_{\text {ch }}$ values, one obtained by integrating the calculated pseudorapidity density distribution from Eq. (5), and another one of the same distribution but being shifted to the left by $\ln (1-\alpha)$, is added to the $N_{\text {ch }}$ value obtained from Eq. (4). Where no pseudorapidity density distributions are available in 
$p p / \bar{p} p$ measurements at $\sqrt{s_{p p}}=3 \varepsilon_{N N}$, the energy balanced limiting fragmentation scaling is applied to reproduce the calculated $\rho(\eta)$ : the measured distribution from a non-central AA collision is shifted by $\left(y_{\text {beam }}-y_{\text {eff }}\right)$, i.e. $\eta \rightarrow \eta+\ln (1-\alpha)$. Then $N_{\text {ch }}$ is calculated as above, by adding to the calculation of Eq. (4) the difference between the integral from the obtained shifted distribution and the measured multiplicity in this non-central AA collision.

Using this ansatz, one finds that the calculated values of $N_{\mathrm{ch}}$ well reproduce the measurements from RHIC, with no deficit in non-central collisions, as shown in Fig. 1(b).

The energy balanced limiting fragmentation scaling provides an explanation of the "puzzle" between the centrality independence of the $N_{\text {part }}$-normalized mean multiplicity and the monotonic decrease of the normalized midrapidity pseudorapidity density with the centrality, as observed at RHIC.

The multiplicity is measured in the full $\eta$-region, so it gets additional contribution from beyond the midrapidity. In the context of the picture proposed here, this contribution is due to the balance between the collision c.m. energy shared by all nucleons of colliding nuclei and the centrality-defined effective energy of the interacting participants. From Fig. 1(b) one can conclude that, in contrast to the RHIC measurements, almost no additional contribution is needed for the PDE calculations of Eq. (4) to describe the LHC data. From this one concludes that in AA collisions at the LHC at $\mathrm{TeV}$ energies the multihadron production obeys a head-on collision regime, at least for the centrality intervals measured so far. This points to apparently different regimes of hadroproduction occurring in AA collisions with $\sqrt{s_{N N}}$ between a few hundred $\mathrm{GeV}$ and $\mathrm{TeV}$ energies. This supports the conclusion made above, which is suggested from the observation of a change of the fit type needed to describe the energy behaviour as soon as the LHC data are included, see Fig. 1(a).

5. Given the obtained agreement between the data and the calculations, and considering the similarity put forward for $\varepsilon_{N N}$ and $\sqrt{s_{N N}}$, one would expect the measured centrality data at $\varepsilon_{N N}$ to follow the $\sqrt{s_{N N}}$ dependence of the mean multiplicity in the most central AA collisions. In Fig. 1(a), the measurements of the charged particle mean multiplicity of head-on AA collisions are added by the centrality measurements by the PHOBOS [10] and the ALICE [8, 17] experiments (Fig. 1(b)) where the centrality data are plotted as a function of $\varepsilon_{N N}$. Due to the above finding of the energy balanced limiting fragmentation scaling these data are plotted by subtracting the energy balanced contribution. From Fig. 1(a), one concludes that effective-energy dependence of the centrality data complements the c.m. energy behaviour of the head-on collision data.

To better trace the similarity between the head-on collision and centrality data, we fit the $\varepsilon_{N N^{-}}$ dependence of the centrality data by the hybrid and the power-law functions, similarly to the head-on collisions. The fits agree well with the same type of fits to the head-on collision data in the entire available energy range.

As soon as the hybrid fit for the head-on collision data and the fit to the centrality data show slightly different increase with c.m. energy, the predictions of the two fits are averaged. Hence, the mean multiplicity $2 N_{\mathrm{ch}} / N_{\text {part }}$ value is predicted to be about 128 with $5 \%$ uncertainty in the most central AA collisions at $\sqrt{s_{N N}}=5.13 \mathrm{TeV}$. The prediction is shown by the right-inclined hatched area in Fig. 1(a). In addition, the fit-averaged prediction based on $p p$ collisions at $\sqrt{s_{p p}}=13 \mathrm{TeV}$, recalculated within the PDE approach, is shown in Fig. 1(a) as the left-inclined hatched area.

Similarly to the existing data on the mean multiplicity $N_{\text {part }}$-dependence, the head-on data hybrid fit is used to make the predictions for the centrality dependence at $\sqrt{s_{N N}}=5.13 \mathrm{TeV}$. The predictions are shown by the dashed-dotted line in Fig. 1(b), where the centrality and $N_{\text {part }}$ values are taken from the $2.76 \mathrm{TeV}$ data. The expectations show an increase of the mean multiplicity with $N_{\text {part }}$ (decrease with centrality) from about 82 to about 128 . The increase looks to be slightly faster than at $\sqrt{s_{N N}}=2.76 \mathrm{TeV}$, especially for the peripheral region. One can see that, except a couple of points 
from peripheral collisions, the predictions are well reproduced by the LHC data, when the latter are scaled by a factor of 1.4 .

6. In this report, we present our recent results of the study [1] of the energy and centrality dependences of the mean multiplicity by extending the earlier energy-dependence analysis $[3,4]$ above the RHIC energies and adding to that the centrality dependence study. In the entire available $\sqrt{s_{N N}}$ range of about a few $\mathrm{TeV}$, the energy dependence of the multiplicity in head-on collisions is found to be well described by the calculations performed within the the approach of the dissipating energy of quark participants. Meanwhile, depending on the data sample, the calculations are found either to describe the measured centrality dependence or to show some deviation between the calculations and the data. For the RHIC data, the deficit in the predictions is observed for non-central collisions so that the predictions do not follow a constancy with the centrality as it is observed at RHIC. The LHC mean multiplicity centrality dependence, however, is found to be well described by the calculations including the increase towards the most central collisions.

To clarify the observations, the pseudorapidity density distribution measured in AA collisions are calculated in the framework of the approach considered here. The energy balanced limiting fragmentation scaling is introduced based on assumption of the similarity of the fragmentation region of the measured distribution in the beam rest frame and that determined from the calculations by using the effective energy. The revealed scaling allows us to reproduce the pseudorapidity distributions independently of the centrality of collisions and then to correctly describe the centrality independence of the mean multiplicity measured at RHIC. Moreover, this finding provides a solution to the RHIC "puzzle" of the difference between the centrality independence of the mean multiplicity vs. the monotonic decrease of the midrapidity pseudorapidity density with the increase of centrality. The mean multiplicity is shown to get a fraction of additional contribution to account for the balance between the collision c.m. energy shared by all nucleons and the effective energy of the participants. However, the midrapidity pseudorapidity density is fully defined by the effective energy of colliding participants.

Given the calculations made in the context of the proposed approach are considering central collisions of nuclei, an agreement between the calculations and the LHC data indicates that at TeV energies the collisions seem to present head-on collisions of the participants at the c.m. energy of the scale of the effective energy. Then, no energy-balanced additional contribution is required even for relatively small number of participants at $\mathrm{TeV}$ energies.

Based on the above findings, the complementarity of the head-on collisions and the centrality data is shown resulting in the similar energy behaviour of the mean multiplicity measurements as soon as the data are considered in terms of the effective energy. A departure of the c.m. energy dependence of the data from the logarithmic behaviour to the power-law one observes at the region of $1 \mathrm{TeV}$ suggests a transition to a new regime in AA collisions at $\mathrm{TeV}$ energies. This is in accordance with the change of the multiplicity dependence on centrality which also indicates a possible change of the regime of multihadron production as one moves from RHIC to LHC. In contrast to heavy ions, no change in multihadron production in $p p$ collisions is expected up to the foreseen LHC energy.

Based on the fits obtained, the predictions are made for the charged particle mean multiplicity in head-on AA and $p p$ collisions at the LHC. Within the obtained complementarity of head-on collisions and centrality data, the predictions are made as well for the mean multiplicity centrality dependence.

\section{Acknowledgements}

The authors are grateful to the XLV International Symposium on Multiparticle Dynamics (ISMD-2015) organizers for kind invitation, warm hospitality and financial support. The work of Alexander Sakharov is partially supported by the US National Science Foundation under Grants No.PHY-1205376 and No.PHY-1402964. 


\section{References}

[1] E.K.G. Sarkisyan, A.N. Mishra, R. Sahoo, A.S. Sakharov, arXiv:1506.09080 [hep-ph]

[2] A.N. Mishra, R. Sahoo, E.K.G. Sarkisyan, A.S. Sakharov, Eur. Phys. J. C 74 (2014) 3147

[3] E.K.G. Sarkisyan, A.S. Sakharov, Eur. Phys. J. C 70 (2010) 533

[4] E.K.G. Sarkisyan, A.S. Sakharov, AIP Conf. Proc. 828 (2006) 35

[5] L.D. Landau, Izv. Akad. Nauk: Ser. Fiz. 17 (1953) 51. English transl.: Collected Papers of L. D. Landau, ed. by D. Ter-Haarp (Pergamon, Oxford, 1965), p. 569

[6] For review, see: V.V. Anisovich, N.M. Kobrinsky, J. Nyiri, Yu.M. Shabelsky, Quark Model and High Energy Collisions (World Scientific, Singapore, 2004)

[7] See e.g. R. Rougny (for the CMS Collab.), Nucl. Phys. B (Proc. Suppl.) 207-208 (2010) 29

[8] ALICE Collab., E. Abbas et al., Phys. Lett. B 726 (2013) 610

[9] PHOBOS Collab., B.B. Back et al., Nucl. Phys. A 757 (2005) 28

[10] B. Alver et al., Phys. Rev. C 83 (2011) 024913

[11] NA49 Collab., S.V. Afanasiev et al., Phys. Rev. C 66 (2002) 054902

[12] E895 Collab.: J.L. Klay, PhD Thesis (U.C. Davis, 2001), see [9]

[13] HADES Collab., G. Agakishev et al., Eur. Phys. J. A 40 (2009) 45

[14] J.F. Grosse-Oetringhaus, K. Reygers, J. Phys. G 37 (2010) 083001

[15] The Review of Particle Physics, K.A. Olive et al. (Particle Data Group), Chin. Phys. C 38, 090001 (2014).

[16] CMS Collab., S. Chatrchyan et al., J. High Energy Phys. 08 (2011) 141

[17] A. Toia (for the ALICE Collab.), J. Phys. G 38 (2011) 124007

[18] LHCb Collab., R. Aaij et al., Eur. Phys. J. C 72 (2012) 1947

[19] TOTEM Collab.., G. Antchev et al., Europhys. Lett. 98 (2012) 31002

[20] W. Kittel, E.A. De Wolf, Soft Multihadron Dynamics (World Scientific, Singapore, 2005)

[21] J. Benecke, T.T. Chou, C.N. Yang, E. Yen, Phys. Rev. 188 (1969) 2159 